\title{
Spectra of bulge stars with known abundance ratios for population synthesis
}

\author{
B. Barbuy ${ }^{1}$, A. Alves-Brito ${ }^{1}$, M. Zoccali ${ }^{2}$, D. Minniti ${ }^{2}$, V. Hill ${ }^{3}$, \\ A. Lecureur ${ }^{3}$, A. Gómez ${ }^{3}$, S. Ortolani ${ }^{4}$, A. Renzini ${ }^{5}$, \\ P. Coelho ${ }^{1}$ and L. Sodré ${ }^{1}$ \\ ${ }^{1}$ IAG-USP, Rua do Matão 1226, 05508-900 São Paulo, Brazil \\ email: barbuy,abrito@astro.iag.usp.br \\ ${ }^{2}$ PUC-Chile, Casilla 306, Santiago 22, Chile \\ email:mzoccali,dante@astro.puc.cl \\ ${ }^{3}$ Observatoire de Paris-Meudon, 92195 Meudon Cedex, France \\ email: Vanessa.Hill@obspm.fr, Aurelie.Lecureur@obspm.fr, Anita.Gomez@obspm.fr \\ ${ }^{4}$ Università di Padova, Dipartimento di Astronomia, Vicolo dell'Osservatorio 5, I-35122 \\ Padova, Italy, \\ email: sergio.ortolani@unipd.it \\ ${ }^{5}$ Osservatorio Astronomico di Padova, Vicolo dell'Osservatorio 2, I-35122 Padova, Italy \\ email: alvio.renzini@oapd.inaf.it
}

\begin{abstract}
Spectra in the range 4000-7000 $\AA$ were obtained for a sample of bulge stars using the GMOS-Gemini low resolution spectrograph. The sample stars were selected from a VLTFLAMES project for the observation of 1000 bulge stars, for which abundance ratios have been derived. Our aim is to study old stellar populations in external galaxies.
\end{abstract}

Keywords. bulge, population synthesis, spectroscopy

\section{Introduction}

We are carrying out a spectroscopic study of 1000 bulge stars using VLT-FLAMES, and among these, 55 stars were also observed with UVES. The first results on the UVES spectra were presented in Zoccali et al. (2006) and Lecureur et al. (2007).

Our main aims are the derivation of metallicity distribution of the bulge, and the determination of abundance ratios in order to better understand the formation of the Galactic bulge. In particular, Kormendy \& Kennicutt (2004) have classified as true bulges those of $\mathrm{Sa}$ and $\mathrm{Sb}$ galaxies, and as pseudo-bulges (formed from dynamical evolution of bars) those of Sc galaxies. We would like to find evidences, to decide if the Milky Way being an Sbc galaxy, has a true bulge or a pseudo-bulge.

We selected stars at one magnitude above the Horizontal Branch (HB), such as to have bright enough stars, and at the same time avoiding the cool Red giant branch (RGB) tip stars where $\mathrm{TiO}$ bands may mask lines of different elements that we intended to measure (see for example Coelho et al. 2001 showing the intensity of $\mathrm{TiO}$ bands in bulge giants). The fields targetted in the VLT-FLAMES project were the Baade's Window $\left(l=1.14^{\circ}\right.$, $\left.\mathrm{b}=-4.2^{\circ}\right)$, field at $-6^{\circ}\left(\mathrm{l}=0.2^{\circ}, \mathrm{b}=-6^{\circ}\right)$, Blanco field $\left(\mathrm{l}=0^{\circ}, \mathrm{b}=-12^{\circ}\right)$, and the field of NGC6553 $\left(\mathrm{l}=5.2^{\circ}, \mathrm{b}=-3^{\circ}\right)$ (see also Zoccali et al., this conference).

The latter field around NGC6553 was targetted for observations with GMOS-Gemini, to obtain low resolution spectra. 


\section{Results from the VLT survey}

The 55 stars observed with UVES were analysed, and abundances of oxygen, magnesium, sodium and aluminum were so far derived. Zoccali et al. (2006) have shown that the oxygen abundance in bulge stars is higher than in the thick disk, which is higher than in the thin disk, using results from Bensby et al. (2004). Lecureur et al. (2007) found that magnesium is also overabundant, confirming the conclusions based on the oxygen abundances. The conclusions are, essentially, that the bulge formed fast, it is old, and older than the thick disk and thin disk. This would indicate that the bulge of the Galactic bulge did not form from secular evolution of a bar.

$\mathrm{Na}$ and $\mathrm{Al}$ are also overabundant with respect to thick and thin disks (Lecureur et al. 2007).

\section{GMOS spectra}

Spectra of the field of NGC $6553\left(1=5.2^{\circ} ; \mathrm{b}=-3^{\circ}\right)$ were obtained with the Gemini Multi-Object Spectrograph (GMOS) on the 8m Gemini South telescope on May 22, 2006, as part of GEMINI Program GS-2006A-Q-41. Three GMOS masks were designed for the spectroscopy. The spectra were obtained as 3 individual exposures with a total exposure time of 300s each. We also used the R400 grating and a slit width of 1 arcsec, achieving a spectral resolution $\mathrm{R} \sim 2000$ at $6500 \AA$ or, approximately, $\Delta \lambda \sim 3 \AA$ and covering the range 4000 to $8000 \AA$. However, the wavelength range varies from star to star due to different slit distances from the bisector of the mask. At wavelengths redder than $7000 \AA$ the spectra show fringing and telluric lines. We believe that corrections will not solve fully the distortions, and in particular since these spectra are to be used as templates, we consider that is only useful in the range 4000-7000 $\AA$.

The data were reduced using the GEMINI GMOS Data Reduction Script which is part of the IRAF package. Bias frames, flat-fields and $\mathrm{CuAr}$ images were taken as part of the GEMINI base calibrations. The reduction process comprised bias-subtraction and flat-fielding, using Gcal flats which were previously co-added and normalized. Following, spectra were cosmic rays cleaned, wavelength calibrated with solutions obtained from the CuAr arc exposures - which provided typical residual of $0.2 \AA$ - sky-subtracted and finally extracted. The flux calibration was not performed yet.

About 104 stars were observed, among which only 10 in common with FLAMES, due to a difference in field size, from $25^{\prime}$ with FLAMES to over $5^{\prime} .5$ with GMOS field. Given the large sample obtained with FLAMES, we should be able to assume that the presently observed sample has the same mean abundance ratios.

In Figs. 1 and 2 is shown the spectrum of a star also observed with FLAMES. In Fig. 1 is shown the full spectrum, whereas in Fig. 2 is shown a zoomed portion of the spectrum.

\section{Conclusions}

We observed 1000 bulge stars with VLT-FLAMES and 55 of them were also observed with UVES. In the field around NGC 6553 we observed 104 stars using GMOS-Gemini. We intend to form a library of bulge stars, for population synthesis.

The results from Zoccali et al. (2006) and Lecureur et al. (2007) have shown that the Milky Way has an old bulge, characterized by high $[\mathrm{O}, \mathrm{Mg} / \mathrm{Fe}] \mathrm{ratios}$, and older than the thick disk.

The knowledge of the abundance ratios, derived from the high resolution spectra, is clearly important in the use of spectra for population synthesis. With GMOS we intend 


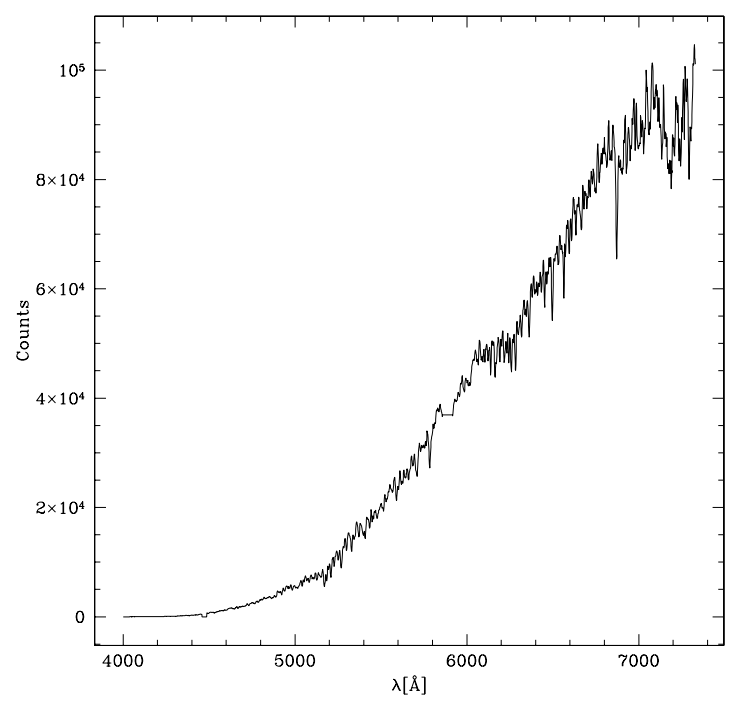

Figure 1. GMOS spectrum of a $V=16.26$ giant.

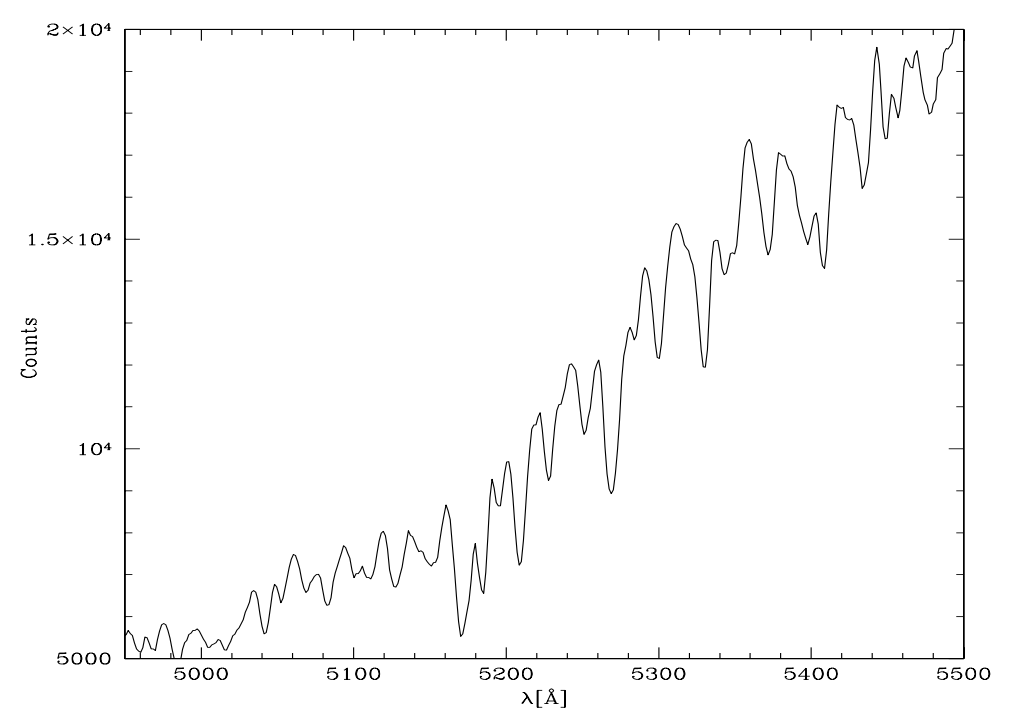

Figure 2. Zoom of same GMOS spectrum of Fig. 3.

to pursue observations in order to have a better coverage of evolutionary stages. We also intend to extend the library to the red and infrared in the future.

\section{Acknowledgements}

BB, AA and LS acknowledge grants from CNPq and Fapesp. DM and MZ acknowledge grants from FONDAP Center for Astrophysics 15010003. This work is partially based on observations obtained at the Gemini Observatory, which is operated by AURA, under a cooperative agreement with USA, Canada, UK, Australia, Argentina, Brazil. 


\section{References}

Bensby, T., Feltzing, S., \& Lundström, I. 2004, A\&A, 415, 155

Coelho, P., Barbuy, B., Perrin, M.-N., Idiart, T., Schiavon, R.P., Ortolani, S., \& Bica, E. 2001, $A \mathscr{E} A, 376,136$

Lecureur, A., Zoccali, M., Hill, V., Barbuy, B., Goméz, A., Minniti, D., Ortolani, S., \& Renzini, A. 2006, $A \& A$, in press (astro-ph/10346)

Kormendy, J., \& Kennicutt, R.C. 2004, ARA\&A, 42, 603

Zoccali, M., Lecureur, A., Barbuy, B., Hill, V., Renzini, A., Minniti, D., Goméz, A., \& Ortolani, S. 2006, A\&A 457, L1

\section{Discussion}

Rose: Can you trust the comparison between abundances of your bulge giants with the Bensby et al. sample of thin and thick disk dwarfs? Do you consider possible systematic discrepancies between giants and dwarfs?

BARBUY: We have used their same atomic parameters in order to minimize possible discrepancies.

PELETIER: What is the temperature range of your sample stars?

BARBUY: For the FLAMES stars it is about 4200-4800 K. But the surrounding stars observed with GMOS should show a broader range in luminosities and temperatures. We also intend to complete data with further observations.

Mouhcine: Do you intend to observe these stars in the infrared?

BARBUY: It would be useful.

Gustafsson: Concerning this issue on consistency between dwarfs and giants, I think that it could be checked empirically, using cluster or visual binaries. I know of a few such tests, e.g., using the Hyades, which I think verified the consistency, but in view of the significance of this issue, maybe one should repeat these tests with modern data and analyses.

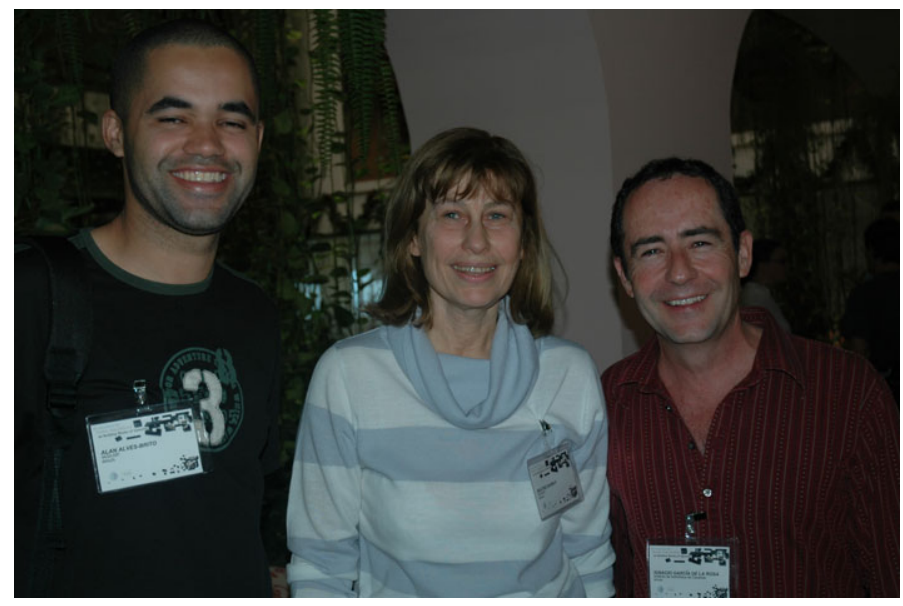

The speaker (middle) with Alan Alves-Brito (left) and Ignacio Garcia de la Rosa. 